\title{
Finitary ideals of direct products in quantales
}

C. Nkuimi-Jugnia

Department of mathematics

University of Yaounde I

Faculty of Sciences

Cameroon

nkuimi@yahoo.co.uk

\begin{abstract}
In this paper, given two quantales non necessary with identity. We investigate the ideals, prime ideals, radical ideals, primary ideals, and maximal ideals of the direct product. Unlike the case where those quantale are unital, an ideal (or primary ideal, or maximal ideal) of the their direct product need not be a sub-product (Lemma 3.1) of ideals. The Theorem 4.2 extends the result on [3] for the product of two quantales.

Keywords- quantale, primness, maximality ideal
\end{abstract}

\section{Introduction}

The notion of quantale, which designates a complete lattice equipped with an associative binary multiplication distributing over arbitrary joins, appears in various areas of mathematics-in quantaloid theory, in non classical logic as completion of the Lindebaum algebra, and in different representations of the spectrum of a $C^{*}$ algebra as many-valued and non commutative topologies. To put it briefly, its importance is no longer to be demonstrated. Quantales are ringlike structures in that they share with rings the common fact that while as rings are semi groups in the tensor category of abelian groups, so quantales are semi groups in the tensor category of sup-lattices. In 2008 Anderson and Kintsinger [1] characterized prime ideals, radical ideals, primary ideals and maximal ideals of $R \times S$ where $R$ and $S$ are commutative rings. In 2009, D. Anderson and V. Camillo [2] given exposition of Goursat's lemma which describes the sub groups of a direct product of two groups. A ring version giving the sub rings and ideals of a direct product of two rings is also given. In this paper we prove some results which are proved by Anderson and Kintsinger in reference [1]. Let us recall some definitions that exist in the literature.

\footnotetext{
${ }^{0}$ ReCiC-Revista de Ciéncia de Computao
}

\author{
P. Pankiti \\ Department of mathematics \\ University of Yaounde I \\ Faculty of Sciences \\ Cameroon \\ pankiti@yahoo.fr
}

\section{BASIC ON QUANTALES}

Definition 2.1. A quantale is a join complete lattice $Q$ with an associative binary operation $\circ: Q \times Q \rightarrow$ $Q$, called its multiplication, satisfying a distributive property such that for all elements $x$ and $y_{i}$ of $Q$, for all $i$ in a set of indexed family $I$, we have the following identities: $x \circ\left(\bigvee y_{i}\right)=\bigvee\left(x \circ y_{i}\right)$ and $\left(\bigvee y_{i}\right) \circ x=\bigvee\left(y_{i} \circ x\right)$.

A quantale is unital if it has an identity element $e$ for its multiplication: $x \circ e=x=e \circ x$, for all $x$ in $Q$. In this case, the quantale is naturally a monoid with respect to its multiplication.

The largest element of $Q$ is denoted by $T$ and the smallest by $\perp$. An element $a$ is called left-sided if $\top \circ a \leq a$, and it called right sided if $a \circ \top \leq a$ and two-sided if it is left-sided and right-sided. A commutative quantale is a quantale whose multiplication is commutative.

Given an ordered set $(Q, \leq)$ the downward closure of an element $x$, denoted by $\downarrow x$ is defined by: $\downarrow x=\{l \in Q: l \leq x\}$. If $Q_{1}$ and $Q_{2}$ are two quantales, then the binary product $Q_{1} \times Q_{2}$ is a quantale where the supremum and the multiplication are defined component by component.

Definition 2.2. [3] A subset $I$ of a quantale $Q$ is called a finitary left ideal of the quantale $Q$ provided that the following conditions are satisfy.

1. For all elements $a$ and $b$ of $I$, the supremum $a \vee b$ is an element of $I$.

2. For all element $x$ of I and $y$ an element of $Q$ where $y \leq x$, we have $y$ is an element of $I$.

3. For all element $x$ of $I$ and all element $q$ of $Q$, we have $q \circ x$ is an element of $I$.

Right ideal is defined in a similar way, replace 3 ) by 3')

3' : For all element $x$ of $I$ and all element $q$ of $Q$, we have $x \circ q$ is an element of $I$.

The ideal $I$ is a two-sided ideal or, simply, an ideal if it is both a left and a right ideal. 
The definition of ideal that we use in this paper is that used by Bergamaschi and Santiago (see [3]) and the paper of Shaoul Liang (see [7]). In all our work, ideal finitaire will be replaced by ideal itself.

As we work only finitaries ideals, they will simply be called ideals, left ideals or right ideals.

For all quantales $Q_{1}$ and $Q_{2}$, non necessary with identity, we investigate the ideals, prime ideals, primary ideals, and maximal ideals of the quantale $Q_{1} \times Q_{2}$. Unlike the case where the quantales $Q_{1}$ and $Q_{2}$ have are unitary, an ideal (or primary ideal, or maximal ideal) of $Q_{1} \times Q_{2}$ need not be a 'sub product' $I \times J$ of ideals. We show that for a quantale $Q_{1}$, for each commutative quantale $Q_{2}$ every ideal (or primary ideal, or maximal ideal) is a sub-product if and only if $Q$ is an e-quantale (that is, for $q \in Q$, there exists an element $e_{q} \in Q$ satisfy the identity $\left.e_{q} \circ q=q\right)$.

An arbitrary intersection of left ideals (resp right ideals, resp. ideal) of a quantale is again a left ideal (resp. right ideal, resp. ideal).

It should observed that in this case, $T \circ \bigvee S$ is a left-sided element where $S$ is a subset of a quantale $Q$. Thus the following result.

Lemma 2.3. For any left-sided element $x$ of a quantale $Q$, the down-set $\downarrow x$ is an ideal with biggest element $x$.

Proof. Let $x$ be an left-sided element of $Q$; the subset $\downarrow x$ is closed under binary supremum and it is closed immediate. Now if $q$ and $l$ are elements of $Q$ and $l \leq x$, then $q \circ l \leq q \circ x \leq \top \circ x \leq x$. The last inequality comes from that $x$ is left-sided element.

Given a subset $S$ of a quantale $Q$, the least left ideal containing $S$, which we denote by $\langle S\rangle_{l}$ is called the left ideal generated by $S$. In particular the left ideal generated by a empty set is $\{\perp\}$ where $\perp$ is the least element of $Q$. We also denote by $\langle S\rangle_{r}($ resp. $\langle S\rangle)$ the right ideal (resp. ideal) generated by $S$.

If $K$ and $J$ are two sub-sets of a quantale $Q$, we define the subset $K \circ J$ by the set $K \circ J:=\{k \circ j$ : $k \in K, j \in J\}$ and the subset $K \bigvee J$ by the following set: $K \bigvee J:=\{k \bigvee j: k \in K, j \in J\}$.

The next lemma is proved by Qingjun Luo and Guojun Wang.

Lemma 2.4. [6] For any quantale $Q$ the following properties hold.

(i) For any subset $S$ of $Q$; the ideal generated by $S$ is given by the set $\langle S\rangle=\left\{x \in Q: x \leq \bigvee_{i=1}^{n} a_{i}\right.$ for some positive integer $n, a_{i} \in S \cup(Q \circ S) \cup(S \circ Q) \cup$ $(Q \circ S \circ Q)\}$. In particular, for each element $a$ of $Q$, $\langle a\rangle=\left\{x \in Q: x \leq \bigvee_{i=1}^{n} a_{i}\right.$ for all positive integer $n$, $\left.a_{i} \in\{a\} \cup(Q \circ a) \cup(a \circ Q) \cup(Q \circ a \circ Q)\right\}$. (ii) The ideals of $Q$ are precisely the sets $\langle a\rangle$ with $a$ be an element of $Q$ satisfies $\bigvee_{i=1}^{n} a_{i} \leq a$ for some positive integer $n, a_{i} \in\{a\} \cup(Q \circ a) \cup(a \circ Q) \cup(Q \circ a \circ Q)$.

The proof of the next lemma is similar, we state it.

Lemma 2.5. Let $S$ be a non empty subset of a quantale $Q$.

(i) The left ideal of $Q$ generated by $S$ is given by $\langle S\rangle_{l}=\left\{x \in Q: x \leq \bigvee_{i=1}^{n} a_{i}\right.$ for some positive integer $\left.n, a_{i} \in S \cup(Q \circ S) \cup(Q \circ S \circ Q)\right\}$.

(ii) The right ideal of $Q$ generated by $S$ is given by $\langle S\rangle_{r}=\left\{x \in Q: x \leq \bigvee_{i=1}^{n} a_{i}\right.$ for some positive integer $\left.n, a_{i} \in S \cup(S \circ Q) \cup(Q \circ S \circ Q)\right\}$.

Lemma 2.6. [3] For all element $a$ of a quantale $Q$, the following inclusion hols: $\langle a\rangle \circ Q \subseteq\langle a\rangle$. If there exists an unit $e$ in $Q$, then $\langle a\rangle \circ Q=\langle a\rangle$.

Definition 2.7. $\quad$ A proper ideal $M$ of a quantale $Q$ is said to be maximal if for each ideal $K$ of $Q$ such that the inclusions $M \subseteq K \subsetneq Q$ are holds, we have the equality $K=M$.

- An ideal $J$ of a quantale $Q$ is said to be completely prime (respectively completely semiprime) if for each elements $x$ and $y$ of $Q$, the element $x \circ y$ is in $J$ implies that $x$ is and element of $J$ or $y$ is an element of $J$ (respectively the element $x \circ x$ is an element of $J$ implies that $x$ is an element of $J$ ).

- An ideal $P$ in a quantale $Q$ is said to be prime (respectively semi-prime) if $P$ for each Ideals $K$ and $J$ of $Q$, the ideal $K \circ J$ satisfy $K \circ J \subseteq P$ implies that $K$ is a subset of $P$ or $J$ is a subset of $P$ (respectively the ideal $K \circ K$ is a subset of $P$ implies that $K$ is a subset of $P$ ).

It is proved in reference [3] that every completely prime ideal of a quantale $Q$ is prime, and if $Q$ is commutative quantale, we have the equivalence of the two notions.

If $Q_{1}$ and $Q_{2}$ are two quantales, a morphism from $Q_{1}$ to $Q_{2}$ is an arrow $f$ defined from $Q_{1}$ to $Q_{2}$ such that $f(x \circ y)=f(x) \circ f(y)$ and $f\left(\bigvee x_{i}\right)=\bigvee f\left(x_{i}\right)$, for all elements $x, y$ and $x_{i}$ of $Q$, where $i$ is element of an indexed family $I$. The collection of quantales and morphisms of quantales form a category, denoted by Quant.

For example, if $R$ is a ring, let $\operatorname{Id}(R)$ the collection of all left ideals of $R ; \operatorname{Id}(R)$ has a structure of quantale where the operations $\bigvee$ and multiplication $\circ$ are defined as follows. $\bigvee K_{i}=\left\langle\cup K_{i}\right\rangle$, the ideal generated by the union of the family $\left\{K_{i}: i \in I\right\}$ and $K \circ J=\{k . j$, where $k \in K$ and $j \in J\}$. Let $Q_{3}$ the set of three elements $\{0, a, 1\}$ with order $0 \leq a \leq 1$; 
$Q$ is a quantale where the multiplication is given by the following table.

\begin{tabular}{|c|c|c|c|}
\hline$\circ$ & 0 & $\mathrm{a}$ & 1 \\
\hline 0 & 0 & 0 & 0 \\
\hline $\mathrm{a}$ & 0 & $\mathrm{a}$ & $\mathrm{a}$ \\
\hline 1 & 0 & $\mathrm{a}$ & 1 \\
\hline
\end{tabular}

Example 2.8. Let $\mathcal{M}=(M, *, e)$ be a monoid; the power set $\mathcal{P}(M)$ has the structure of quantale where the suprema is given by the union and the multiplication is defined as follows: for each subsets $A$ and $B$ of $M, A \circ B$ is the set given by $A \circ B=\{a * b: a \in$ $A, b \in B\}$. The identity in this quantale is the subset $\{e\}$. It is clear that if the monoid $\mathcal{M}$ is commutative, then again the quantale $(\mathcal{P}(M), \subseteq, \circ)$ is again commutative. In reference [4] the author call the quantale $(\mathcal{P}(M), \subseteq, \circ)$ the relational quantale. It is proved in reference [4] that each quantale is isomorphic to a relational quantale.

\section{Ideals of product of two quan- tales}

In a quantale $Q$, it is away that for all element $q$ of $Q$, we have $q \circ \perp=\perp \circ q=\perp$; the least element $\perp$ is the empty suprema, so $q \circ \perp=q \circ \bigvee \emptyset=\bigvee_{x \in \emptyset}(q \circ x)=$ $\bigvee \emptyset=\perp$ We next turn to direct product of quantales and their ideals. If $Q_{1}$ and $Q_{2}$ are quantales, and $I_{1}$ and $I_{2}$ are ideals of $Q_{1}$ and $Q_{2}$ respectively, then $I_{1} \times I_{2}$ is an ideal of the quantale $Q_{1} \times Q_{2}$. Similar statements hold for right and left ideal ideals. If $Q_{1}$ and $Q_{2}$ have an identity, then every ideal (right, left or two-sided) has this form. This is our next lemma.

Lemma 3.1. For any unital quantales $Q_{1}$ and $Q_{2}$, every left ideal of the quantale $Q_{1} \times Q_{2}$ is a subproduct of left ideals, that is the ideal of $Q_{1} \times Q_{2}$ has the form $K_{1} \times K_{2}$, where $K_{i}$ is a left ideal of $Q_{i}$, for $i=1$ or $i=2$.

Proof. First, if $K_{i}$ is an ideal of $Q_{i}$, then $K_{1} \times K_{2}$ is clearly an ideal of $Q_{1} \times Q_{2}$. Secondly, let $K$ be an ideal of the quantale $Q_{1} \times Q_{2}$; we will prove that $K$ is a sub-product. Put $K_{1}=\left\{a \in Q_{1}:(a, \perp) \in K\right\}$ and $K_{2}=\left\{b \in Q_{2}:(\perp, b) \in K\right\}$. We have $(\perp, \perp) \in K$, then $\perp \in K_{1}$. Now, let $a \in K_{1}$ and $a^{\prime} \in Q_{1}$; We have $\left(a^{\prime}, \perp\right) \circ(a, \perp)=\left(a^{\prime} \circ a, \perp\right)$ is an element of $K$, because $(a, \perp) \in K$ and $K$ is an ideal of $Q_{1} \times Q_{2}$. So $a \circ a^{\prime}$ is an element of $K_{1}$. Let $a_{1}$, and $a_{2}$ be two elements of $K_{1} ;\left(a_{i}, \perp\right)$ are in $K$ for $i=1,2$. Therefore the equality $\left(a_{1}, \perp\right) \vee\left(a_{2}, \perp\right)=\left(a_{1} \vee a_{2}, \perp\right)$ holds and we conclude that $\left(a_{1}, \perp\right) \vee\left(a_{2}, \perp\right)$ is an element of $K$; this implies that $a_{1} \vee a_{2}$ is an element of $K_{1}$. Let $x_{1}$ be an element of $K_{1}$ and let $y_{1}$ be an element of $Q_{1}$ with respect $y_{1} \leq x_{1}$. The element $\left(x_{1}, \perp\right)$ is in $K$; so we have $\left(y_{1}, \perp\right) \leq\left(x_{1}, \perp\right)$ and then $\left(y_{1}, \perp\right)$ is an element of $K$; so $y_{1}$ is an element of $K_{1}$ and we conclude that $K_{1}$ is an ideal; similarly, $K_{2}$ is an ideal of $Q_{2}$. To finish this proof, it is let to check that is $K=K_{1} \times K_{2}$. Let $(a, b)$ an element of $K$; then $(a, \perp)=(e, \perp) \circ(a, b)$ is an element of $K$ and $a$ is an element of $K_{1}$. Similar we prove that $b$ is an element of $K_{2}$. Now, for all element $(a, b)$ of the set $K_{1} \times K_{2},(a, \perp)$ and $(\perp, b)$ are elements of $K_{1}$ and $K_{2}$ respectively; we have the following equality: $(a, b)=(e, \perp) \circ(a, \perp) \vee(\perp, e) \circ(\perp, b)$, and we conclude that $(a, b)$ is an element of $K$. We conclude that $K=K_{1} \times K_{2}$.

Proposition 3.2. Let $Q_{1}$ and $Q_{2}$ be two quantales with least elements denoted by $\perp$; the following conditions are equivalent .

1. Every ideal $K$ of $Q_{1} \times Q_{2}$ is a sub-product.

2. For each element $a$ of $Q_{1}$ and each element $b$ of $Q_{2}$, we have the equality $\langle(a, b)\rangle=\langle a\rangle \times\langle b\rangle$.

3. For each element $a$ of $Q_{1}$ and each element $b$ of $Q_{2}$, the element $(a, \perp),(\perp, b)$ is an element of $\langle(a, b)\rangle$.

Proof. $(1) \Rightarrow(2)$. Assume that every ideal of the quantale $Q_{1} \times Q_{2}$ is a sub-product; let $a$ be an element of $Q_{1}$ and $b$ be an element of $Q_{2}$. We want to show the equality $\langle(a, b)\rangle=\langle a\rangle \times\langle b\rangle$. The subset $\langle(a, b)\rangle$ is an ideal of the quantale $Q_{1} \times Q_{2}$, then by hypothesis it is the form $K_{1} \times K_{2}$ where $K_{i}$ is an ideal of $Q_{i}, i=1,2$; the pair $(a, b)$ is an element of the set $K_{1} \times K_{2}$, then $a$ is an element of $K_{1}$ and $b$ is an element of $K_{2}$, this implies the following inclusions $\langle a\rangle \subseteq K_{1}$ and $\langle b\rangle \subseteq K_{2}$. So the inclusion $\langle a\rangle \times\langle b\rangle \subseteq\langle(a, b)\rangle$ hols. The pair $(a, b)$ is an element of $\langle a\rangle \times\langle b\rangle$ and $\langle a\rangle \times\langle b\rangle$ is an ideal of $Q_{1} \times Q_{2}$, then $\langle(a, b)\rangle \subseteq\langle a\rangle \times\langle b\rangle$, therefore $\langle a\rangle \times\langle b\rangle=\langle(a, b)\rangle$.

$(2) \Rightarrow(3)$. Assume that for each element $a$ of $Q_{1}$ and $b$ an element of $Q_{2}$, the pair is write $\langle(a, b)\rangle=\langle a\rangle \times\langle b\rangle$. Let $a$ be an element of $Q_{1}$ and $b$ be an element of $Q_{2}$; The pair $(a, b)$ is an element of $\langle(a, b)\rangle=\langle a\rangle \times\langle b\rangle$, so $(a, \perp)$ is an element of $\langle a\rangle \times\langle b\rangle$; therefore the pair $(a, \perp)$ is an element of $\langle(a, b)\rangle$.

$(3) \Rightarrow(1)$. Assume that for each element $a$ of the quantale $Q_{1}$ and each element $b$ of the quantale $Q_{2}$, the pairs $(a, \perp)$ and $(\perp, b)$ are elements of $\langle(a, b)\rangle$. Let $K$ be an ideal of the quantale $Q_{1} \times Q_{2}$; put $K_{1}:=\left\{a \in Q_{1}:(a, \perp) \in K\right\}$ and $K_{2}:=\left\{b \in Q_{2}:\right.$ $(\perp, b) \in K\}$. Let $a$ be an element of $K_{1}$ and $a^{\prime}$ be an element of $Q_{1}$ such that $a^{\prime} \leq a$; the pair $(a, \perp)$ is an element of $K$ and $\left(a^{\prime}, \perp\right) \leq(a, \perp)$, then the pair $\left(a^{\prime}, \perp\right)$ is an element of $K$ and $a^{\prime}$ is an element of $K_{1}$. For all pair $(a, b)$ of $K_{1} \times K_{2}, a$ is an element of $K_{1}$ 
and $b$ is an element of $K_{2}$, then the pairs $(a, \perp)$ and $(\perp, b)$ are elements of $K$ and the following equality hols: $(a, b)=(a, \perp) \vee(\perp, b)$ and conclude that the pair $(a, b)$ is an element of $K$; this prove that the set $K_{1} \times K_{2}$ is a subset of $K$. To finish, let $(a, b)$ be an element of $K$; the pairs $(a, \perp)$ and $(\perp, b)$ are elements of $\langle(a, b)\rangle$, so $a$ is element of $K_{1}$ and $b$ is element of $K_{2}$ and we conclude that the equality $K=K_{1} \times K_{2}$ holds.

Of course Lemma 3.1 may fail if $Q_{1}$ and $Q_{2}$ do not have an identity. For example, we next give a partial converse to Lemma 3.1. Let us call a quantale a left e-quantale if for each element $q$ of $Q$ there exists an element $e_{q}$ of $Q$ depending of $q$ with $e_{q} \circ q=q$. Note that $Q$ is a left e-quantale if and only if $Q \circ I=I$ for each left ideal $I$ of $Q$.

Theorem 3.3. For a quantale $Q_{1}$, with top element $\top$ and bottom element $\perp$, satisfies for all elements $a$ and $b$ of $Q_{1}, a \leq b \circ a$ implies that $a=b \circ a$. The following conditions are equivalent in $Q_{1}$.

(1) The quantale $Q$ is a left e-quantale (that is, for each element $q$ of $Q$, there exists an element $e_{q}$ of $Q_{1}$ with $\left.e_{q} \circ q=q\right)$.

(2) For each unital quantale $Q_{2}$, each left ideal of $Q_{1} \times Q_{2}$ is a sub-product.

(3) For all natural number $n$ such that $n \geq 2$, each left ideal of the quantale $Q_{1}^{n}$ has the form $K_{1} \times \ldots \times K_{n}$ where $K_{i}$ is an left ideal of $Q_{1}$ for all $i$ element of $\{1,2, \ldots, n\}$.

(4) Every left ideal of the quantale $Q_{1} \times Q_{1}$ is a subproduct.

Proof. $(1) \Rightarrow(2)$. Assume that $Q_{1}$ is an e-quantale and let $Q_{2}$ be an unital unital quantale denoted $e$. Let $K$ be an ideal of $Q_{1} \times Q_{2}$. Put $K_{1}=\left\{a \in Q_{1}\right.$ : $(a, \perp) \in K\}$ and $K_{2}=\left\{b \in Q_{2}:(\perp, b) \in K\right\}$; For each pair $(a, b)$ of $K_{1} \times K_{2}, a$ is an element of $K_{1}$ and $b$ is an element $K_{2}$, so $(a, \perp)$ is an element of $K$ and $(\perp, b)$ is an element of $K ;(a, b)=(a, \perp) \vee(\perp, b)$ is an element of $K$ and $K_{1} \times K_{2} \subseteq K$. Now let $(a, b)$ be element of $K$; choose $e_{a}$, element of $Q_{1}$ with $e_{a} \circ a=a$. Then $(a, \perp)=\left(e_{a}, \perp\right) \circ(a, b)$ is an element of $K$; so $a$ is an element of $K_{1}$. And $(\perp, b)=(\perp, e) \circ(a, b)$ is an element of $K$; so $b$ is an element of $K_{2}$.

$(2) \Rightarrow(3)$. By induction on $n$, if $n=2$, apply (2) with $Q_{2}=Q_{1}$. Assume the result for $n-1$ and take $Q^{\prime}$ given by $Q_{2}=Q_{1}^{n-1}$ and conclude.

$(3) \Rightarrow(4)$ : clear.

$(4) \Rightarrow(1)$. Let $q$ be an element of $Q$, then the pair $(q, q)$ is an element of $Q_{1} \times Q_{1}$ and the pair $(q, q)$ is an element of $\langle(q, q)\rangle=\langle q\rangle \times\langle q\rangle$. So the pair $(q, \perp)$ is an element of $\langle q\rangle \times\langle q\rangle$ (use 3.2); there exists a finite family $\left\{a_{i}: 1 \leq i \leq n\right\}$ of elements of $Q \circ q \cup Q \circ q$, where $n$ is a positive entire number, such that $q \leq \bigvee_{i=1}^{n} a_{i}$; so $q \leq\left(\bigvee_{i=1}^{n} b_{i}\right) \circ q$. We conclude that $q=e_{q} \circ q$, where $e_{q}=\bigvee_{i=1}^{n} b_{i}$.

Example 3.4. If $Q_{1}$ is a idempotent quantale (That is $x \circ x=x$ for all element $x$ of $Q_{1}$ ), then $Q$ is an equantale. Every left ideal of $Q_{1} \times Q_{2}$ is a sub-product, for all unital quantale $Q_{2}$.

Example 3.5. Let $Q=\{0, a, b, c, 1\}$ with the order $\leq$, given by $0 \leq a \leq c \leq 1$ and $0 \leq b \leq c \leq 1$ is a quantale where their multiplication is giving by the following table.

\begin{tabular}{|c|c|c|c|c|c|}
\hline$\circ$ & 0 & $\mathrm{a}$ & $\mathrm{b}$ & $\mathrm{c}$ & 1 \\
\hline 0 & 0 & 0 & 0 & 0 & 0 \\
\hline $\mathrm{a}$ & 0 & $\mathrm{a}$ & 0 & $\mathrm{a}$ & $\mathrm{a}$ \\
\hline $\mathrm{b}$ & 0 & 0 & $\mathrm{~b}$ & $\mathrm{~b}$ & $\mathrm{~b}$ \\
\hline $\mathrm{c}$ & 0 & $\mathrm{a}$ & $\mathrm{b}$ & $\mathrm{c}$ & $\mathrm{c}$ \\
\hline 1 & 1 & $\mathrm{a}$ & $\mathrm{b}$ & $\mathrm{c}$ & 1 \\
\hline
\end{tabular}

We see that $Q_{1}$ is an e-quantale, and any (left, right, two-sided) ideal of $Q_{1} \times Q_{2}$, where $Q_{2}$ is a commutative quantale is a sub-product.

We leave it to the reader to define a right equantale and to state versions of theorem 3.3 for right ideals and two-sided ideals. Note that if $\left\{Q_{i}: i \in I\right\}$ is any nonempty family of left e-quantales, then their direct product $\prod_{i \in I} Q_{i}$ with coordinate-wise operations is again a left e-quantale. In particular, an infinite direct product of quantales each having an identity is both a left and right e-quantale, but does not have an identity.

\section{Primeness}

Lemma 4.1. [3] For an ideal $P$ in a quantale $Q$ with unity, the following statements are equivalent:

(1) the ideal $P$ is prime.

(2) For any elements $a$ and $b$ of $Q$, the inclusion $\langle a\rangle \circ\langle b\rangle \subseteq P$ holds implies $\langle a\rangle \subseteq P$ or $\langle b\rangle \subseteq P$.

(3) For any elements $a$ and $b$ of $Q$, the inclusion $a \circ Q \circ b \subseteq P$ holds implies that $a$ is an element of $P$ or $b$ is an element of $P$.

Theorem 4.2. Let $Q_{1}$ and $Q_{2}$ be quantales. Then an ideal $\mathcal{P}$ of the quantale $Q_{1} \times Q_{2}$ is prime if and only if $\mathcal{P}$ has the form $P_{1} \times Q_{2}$ where $P_{1}$ is a prime ideal of $Q_{1}$ or $Q_{1} \times P_{2}$ where $P_{2}$ is a prime ideal of $Q_{2}$.

Proof. $\Rightarrow$ ) Assume that $P_{1}$ is a prime ideal of $Q_{1}$. Let $I$ and $J$ be two ideals of $Q_{1} \times Q_{2}$ such that $I \circ J \subseteq P_{1} \times Q_{2} ; I$ and $J$ are ideals of the quantale $Q_{1} \times Q_{2}$, then $I$ and $J$ have the form $I=I_{1} \times I_{2}$ and $J=J_{1} \times J_{2}$ where $I_{k}$ and $J_{k}$ are ideals of $Q_{k}$ for $k=1,2$. The following inclusions are hold: $I \circ J=$ $\left(I_{1} \times I_{2}\right) \circ\left(J_{1} \times J_{2}\right)=\left(I_{1} \circ J_{1}\right) \times\left(I_{2} \circ J_{2}\right) \subseteq P_{1} \times Q_{2} ;$ 
so $I_{1} \circ J_{1} \subseteq P_{1}$ and $P_{1}$ is a prime ideal. So $I_{1} \subseteq P_{1}$ or $J_{1} \subseteq P_{1}$ and deduce that $I \subseteq P_{1} \times Q_{2}$ or $J \subseteq P_{1} \times Q_{2}$ and conclude that $P_{1} \times Q_{2}$ is a prime ideal. Similarly we prove that if $P_{2}$ is a prime ideal of $Q_{2}$, then $Q_{1} \times P_{2}$ is a prime ideal of $Q_{1} \times Q_{2}$. Conversely, Assume that $\mathcal{P}$ is a prime ideal of $Q_{1} \times Q_{2}$. Denoted by $0=\{\perp\}$ the least ideal of $Q_{1}$ or $Q_{2}$. Now $\left(0 \times Q_{2}\right) \circ(Q \times 0) \subseteq \mathcal{P}$, so either $0 \times Q_{2} \subseteq \mathcal{P}$ or $Q_{1} \times 0 \subseteq \mathcal{P}$. Assume that $Q \times 0 \subseteq \mathcal{P}$. It follows from 3.2 that $\mathcal{P}=Q_{1} \times P_{2}$ for some ideal $P_{2}$ of $Q_{2}$. It is easily checked that $P_{2}$ must be prime. The case where $0 \times Q_{2} \subseteq \mathcal{P}$ is similar.

Similarly we prove the following theorem.

Theorem 4.3. Let $Q_{1}$ and $Q_{2}$ be quantales. Then an ideal $\mathcal{P}$ of $Q_{1} \times Q_{2}$ is semi-prime if and only if $\mathcal{P}$ has the form $P_{1} \times Q_{2}$ where $P_{1}$ is a semi-prime ideal of $Q_{1}$ or $Q_{1} \times P_{2}$ where $P_{2}$ is a semi-prime ideal of $Q_{2}$.

Lemma 4.4. For a commutative quantale $Q_{1}$, the following conditions are equivalent.

1. For each commutative quantale $P$, every maximal ideal of $Q_{1} \times P$ has the form $M \times P$ or $Q_{1} \times N$, where $M$ (respectively $N$ ) is a maximal ideal of $Q_{1}$ (respectively $P$ ).

2. Every maximal ideal of $Q_{1} \times Q_{1}$ has the form $M \times Q_{1}$ or $Q_{1} \times M$ where $M$ is a maximal ideal of $Q_{1}$.

3. Every maximal ideal of $Q_{1} \times Q_{1}$ is a subproduct.

4. For each commutative quantale $P$, every maximal ideal of $Q_{1} \times P$ is a sub-product.

Proof. The implication $1 \Rightarrow 2 \Rightarrow 3 \Rightarrow 4$ are clear. $(4) \Rightarrow(1)$. Let $P$ a commutative quantale and let $\mathcal{M}$ be a maximal ideal of $Q_{1} \times P$; (4) implies that $\mathcal{M}$ is a sub-product, then $\mathcal{M}=M \times N$, where $M$ is an ideal of $Q_{1}$ and an $N$ is an ideal of $P$. We have the inclusion $M \times N \subseteq M \times P$ and the inclusion $M \times N \subseteq Q_{1} \times N$, so $\mathcal{M}$ has the form $M \times P$, where $\mathrm{M}$ is a maximal ideal of $Q_{1}$ or $\mathcal{M}$ has the form $Q_{1} \times N$, where $N$ is a maximal ideal of $P$.

Proposition 4.5. For a commutative unital quantale $Q_{1}$, the following conditions are equivalent.

1. Every maximal ideal of $Q_{1}$ is prime.

2. For each commutative quantale $Q_{2}$, every maximal ideal of $Q_{1} \times Q_{2}$ is prime.

3. Every maximal ideal of $Q_{1} \times Q_{1}$ is prime.
Proof. (1) $\Rightarrow(2)$. Let $\mathcal{M}$ a maximal ideal of $Q_{1} \times Q_{2} ;$ used 3.2 and conclude that $\mathcal{M}$ has the form $M \times Q_{2}$ where $M$ is a maximal ideal of $Q_{1}$; so $M$ maximal and used (1) and conclude that $M$ is prime.

$(2) \Rightarrow(3)$ is clear.

$\left(3 \Rightarrow(1)\right.$. Let $M$ be a maximal ideal of $Q_{1}$; then 3.3 implies that $M \times Q_{1}$ is a maximal ideal of $Q_{1} \times Q_{1}$; (3) implies that $M \times Q_{2}$ is a prime ideal of $Q_{1} \times Q_{1}$. Lemma 3.3 implies that $M$ is prime.

Definition 4.6. For any commutative unitary quantale $Q$ an $I$ an ideal of $Q$, the radical of $I$ is the the denoted by $\operatorname{Rad}(I)$ and defined by $\operatorname{Rad}(I)=:\{a \in$ $Q: a^{n} \in I$ for some $\left.n \in \mathbb{N}\right\}$. Note that for an element $a$ of $Q, a^{n}=a \circ a \cdots \circ a$. An ideal $I$ of $Q$ is said to be radical if $\operatorname{Rad}(I)=I$.

Proposition 4.7. Let $Q$ be a unitary commutative quantale and $I$ be an ideal of $Q$; the following results are hold.

(1) The subset $\operatorname{Rad}(I)$ is an ideal of $Q$ contain $I$.

(2) If $I$ is a prime ideal, then $I$ is radical.

(3) The correspondence $\operatorname{Rad}(-)$ defined on the set of all ideals $Q$ is an idempotent endofunctor.

(4) The correspondence $\operatorname{Rad}(-)$ is a closure operator.

(5) If $I$ is a primary ideal, then the radical of $I$ is a prime ideal of $Q$.

(6) The radical of $I$ is the intersection of all prime ideals of $Q$ containing $I$.

Proof. (1) It is easy with $n=1$ to see that I is a subset of $\operatorname{Rad}(I)$. Let $x$ and $y$ be elements of $\operatorname{Rad}(I), q$ be an element of $Q$. There exists the positive integer $n$ and $m$ such that $x^{n}$ and $y^{m}$ are elements of $I$. We have $(q \circ x)^{n}=q^{n} \circ x^{n}$ is an element of I, so $q \circ x$ is an element of $\operatorname{Rad}(I)$. If $q$ is such that $q \leq x$, so $q^{n} \leq x^{n}$ an conclude that $q$ is an element of $\operatorname{Rad}(I)$. To finish, see that $x^{n+m}$ and $y^{n+m}$ are elements of $I$ and $x^{n+m} \vee y^{n+m}$ is an element of $I$. We remark that $(x \vee y)^{n+m}=x^{n+m} \vee y^{n+m} \vee(x \circ y)^{n+m}$ is an element of $I$ and conclude that $x \vee y$ is an element of $\operatorname{Rad}(I)$. (2) Assume that $I$ is a prime ideal. Let $x$ be an element of $\operatorname{Rad}(I)$ and $n$ be a positive integer number such that $x^{n}$ is an element of $I$. We have $x^{n}=x \circ x^{n-1}$ is an element of $I$. By induction on $n$, we prove that $x$ is an element of $I$ because $I$ is prime.

(3) Let $J$ be an ideal of $Q$ such that $I \subseteq J$; let $x$ be an element of $\operatorname{Rad}(I)$ and $n$ be a positive integer number such that $x^{n}$ is an element of $I$, so $x^{n}$ is an element of $J$ and conclude that $x$ is an element of $\operatorname{Rad}(J)$. Now, let $a$ be an element of $\operatorname{Rad}(\operatorname{Rad}(I))$; there is a positive integer number $m$ such that $a^{m}$ is an element of $\operatorname{Rad}(I)$; there is a positive integer number $n$ such $\left(a^{m}\right)^{n}$ is in $I ;\left(a^{m}\right)^{n}=a^{n m}$ is in $I$ implies that $a$ is an element of $\operatorname{Rad}(I)$, so $\operatorname{Rad}(\operatorname{Rad}(I))=\operatorname{Rad}(I)$.

(4) Follows by (1) and (3). 
(5) Let $a$ and $b$ be two elements of $Q$ such that $a \circ b$ is an element of $\operatorname{Rad}(I)$; so there is a positive number $n$ such that $(a \circ b)^{n}=a^{n} \circ b^{n}$ is an element of $I$, so there is a positive integer $m$ such that $a^{n m}$ is an element of $I$ or $b^{n m}$ is an element of $I$. We say that $a$ or $b$ is an element of $\operatorname{Rad}(I)$.

(6) Let $P$ be a prime ideal of $Q$ containing $I$; If $x$ is an element of $\operatorname{Rad}(I)$ and $n$ be an entire number such that $x^{n}$ is an element of $I$, the element $x^{n}$ is in $P$ and conclude that $x$ is an element of $P$.

It is not hard to show the following proposition.

Proposition 4.8. For each ideals $I$ and $J$ of a commutative quantale $Q, \operatorname{Rad}(I \cap J)=\operatorname{Rad}(I) \cap \operatorname{Rad}(J)$.

Theorem 4.9. Let $P$ and $Q$ be commutative quantales. The radical ideals of $P \times Q$ have the form $I \times J$ where $I$ is a radical ideal of $P$ and $J$ is a radical ideal of $Q$.

Proof. Let $K$ be a radical ideal of $P \times Q$. We may assume that $K$ is a sub-product of the form $I \times J$ where $I$ is an ideal of $P$ and $J$ an ideal of $Q$. So $I$ is an intersection of prime ideals, each of which is a sub-product. So $K=I \times J$ is a sub-product where $I$ and $J$ is either the whole quantale or an intersection of prime ideals. In either case $I$ and $J$ is a radical ideal.

Our next goal is to characterize the commutative quantales $Q$ with the property that for each commutative quantale $P$, every primary ideal of is a subproduct. We need the following lemma.

Lemma 4.10. Let $P$ and $Q$ be commutative quantales.

(1) If $A$ is an ideal with $A \neq Q$ and $\operatorname{Rad}(A)=Q$, then $A$ is primary.

(2) If $K$ is a primary ideal of $P \times Q$ with $\operatorname{Rad}(K) \neq$ $P \times Q$, then either $K=I_{1} \times Q$ where $I_{1}$ is a primary ideal of $P$ or $K=P \times I_{2}$ where $I_{2}$ is a primary ideal of $Q$.

Proof. (1) Assume that $a$ and $b$ are elements of $Q$ such that $a \circ b$ is an element of $A$. Then $\operatorname{Rad}(A)=Q$ gives $b^{n}$ is an element of $A$ for some $n \geq 1$ regardless of whether $a$ is an element of $A$ or not. (2) Now $\operatorname{Rad}(K)$ is a prime ideal of $P \times Q$, so either $\operatorname{Rad}(K)=A \times Q$ where $A$ is a prime ideal of $P$ or $\operatorname{Rad}(K)=P \times B$ where $B$ is a prime ideal of $Q$. Without loss of generality we may assume that $\operatorname{Rad}(K)=A \times Q$ where $A$ is a prime ideal of $P$. Let $x$ be an element of $P-A$; so $(x, \perp)$ is not an element of $\operatorname{Rad}(A)$. Let $q$ be an element of $Q$. Then $(\perp, q) \circ(x, \perp)=(\perp, \perp)$ is an element of $A$ and $(x, \perp)$ is not element of $\operatorname{Rad}(A)$, so $(\perp, q)$ is an element of $A$, since $A$ is primary. Hence $0 \times Q$ is a subset of $A$. So by Proposition $1, Q=A \times Q$ for some ideal $A$ of $P$ which is easily seen to be primary.

\section{Conclusion}

In view of the results we obtained in this paper and those obtained by other authors on quantales, we see a lot of similarities between the category of quantales and the category of rings. We have established that when we give ourselves two unital quantales $Q_{1}$ and $Q_{2}$, the ideals of the direct product $Q_{1} \times Q_{2}$ are subproducts, that is to say of the form $K_{1} \times K_{2}$ where $K_{i}$ is ideal of $Q_{i}$ for $i \in\{1,2\}$. We have also established that the prime ideals (resp. semi-prime ideal, resp maximal ideals) of $Q_{1} \times Q_{2}$ are of the form $P_{1} \times Q_{2}$ or $Q_{1} \times P_{2}$ where $P_{i}$ is a prime ideal (resp. semi prime ideal, resp. maximal ideal) of $Q_{i}$ for $i=1,2$.

\section{References}

[1] D.D.Anderson, and John Kintzinger. Ideals in direct product of commutative rings. Bull. Austral. Math. Soc. 77 (2008), 477-483.

[2] D.D.Anderson, V. Camillo. Subgroups of direct products of groups, ideals and subrings of direct products of rings, and Goursat's lemma. Contemporary Mathematics, volume 480, 2009.

[3] F.B. Bergamaschi, R.H.N. Santiago, A.O. Andrade, Primeness in Quantales, ReCiC-Revista de Ciéncia de Computa.

[4] Carolyn Brown and Doug Gurr Department of Computer Sciences, Chalmers University of Technology, S-41296 Goteborg,Journal of Pure and Applied Algebra 85 (1993) 27-42 North-Holland.

[5] Ciro Russo. Quantales and Their Modules: Projective Objects, Ideals, and Congruences. South American Journal of Logic, Vol. 2, n. 2, pp. 405424, 2016.

[6] Qingjun Luo and Guojun Wang. Roughness and fuzziness in quantales. Information Sciences, 271:14-30, 2014.

[7] Shaolul Liang. The points and diameters of quantales. Open Math, 2018, 16: 636-645 . 